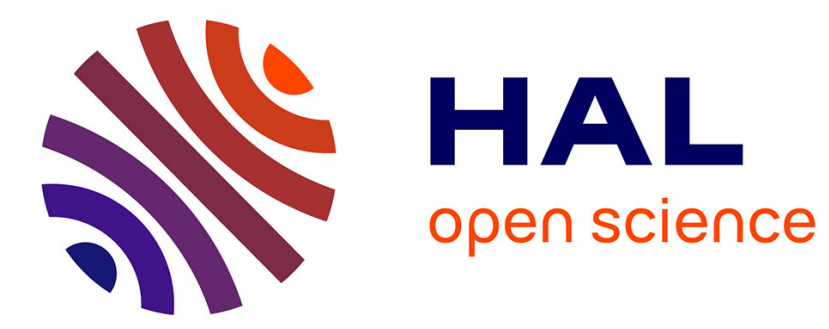

\title{
Garvey-Kelson relations and the new nuclear mass tables
}

\author{
J. Barea, A. Frank, J. Hirsch, P. van Isacker, S. Pittel, V. Velazquez
}

\section{To cite this version:}

J. Barea, A. Frank, J. Hirsch, P. van Isacker, S. Pittel, et al.. Garvey-Kelson relations and the new nuclear mass tables. Physical Review C, 2008, 77, pp.041304(R). 10.1103/PhysRevC.77.041304 . in2p3-00256302

\section{HAL Id: in2p3-00256302 https://hal.in2p3.fr/in2p3-00256302}

Submitted on 15 Feb 2008

HAL is a multi-disciplinary open access archive for the deposit and dissemination of scientific research documents, whether they are published or not. The documents may come from teaching and research institutions in France or abroad, or from public or private research centers.
L'archive ouverte pluridisciplinaire HAL, est destinée au dépôt et à la diffusion de documents scientifiques de niveau recherche, publiés ou non, émanant des établissements d'enseignement et de recherche français ou étrangers, des laboratoires publics ou privés. 


\title{
Garvey-Kelson Relations and the New Nuclear Mass Tables
}

\author{
J. Barea, A. Frank, and J. Hirsch \\ Instituto de Ciencias Nucleares, Universidad Nacional Autónoma de México, \\ Ap. Postal 70-543, 04510 México, D.F. México \\ P. Van Isacker \\ Grand Accélérateur National d'Ions Lourds, CEA/DSM-CNRS/IN2P3, BP 55027, F-14076 Caen Cedex 5, France
}

S. Pittel

Bartol Research Institute and Department of Physics and Astronomy, University of Delaware, Newark, Delaware 19716, USA

V. Velázquez

Departamento de Fúsica, Facultad de Ciencias, Universidad Nacional Autónoma de México, Ap. Postal 70-542, 04510 México, D.F. México

(Dated: January 22, 2008)

\begin{abstract}
We discuss the Garvey-Kelson mass relations in an extended formalism and show how they can be used to test and improve the consistency of the most commonly used mass formulae, in order to achieve more accurate predictions.
\end{abstract}

PACS numbers: 21.10.Dr, 21.30.-x, 21.60.-n, 13.75.Cs

A large number of processes in nuclear physics require for their description an accurate knowledge of nuclear masses. Mass predictions are, for example, a critical element of many calculations required in nuclear astrophysics [1]. Though much progress has been made in measuring the masses of exotic nuclei, theoretical models are still necessary to predict them in regions far from stability [2]. Efforts have typically come from two directions. On the one hand, there has been much work in developing mass formulae with both microscopic and macroscopic input. Alternatively, there have been important advances towards the derivation of masses from a fully microscopic framework.

In this Letter we wish to emphasize the potential use of the Garvey-Kelson (GK) mass relations [3] to provide constraints on the mass formulae that emerge. As we will show, the GK relations lead to masses in extremely close agreement with measured masses throughout most of the periodic table, with errors typically much less than those of mass formulae obtained either from macroscopic/microscopic or purely microscopic considerations. We will also see that most of those mass formulae are not compatible with the GK relations, in the sense that they do not satisfy the GK relations at the same level that the GK relations reproduce the measured data. This is particularly evident when those GK-incompatible mass models are extended to include nuclei outside their fits. The differences between the mass models and the GK relations are such as to suggest that some nuclear correlations are missing from these mass models. To accommodate the missing correlations, we thus suggest the possible use of the GK mass relations in the fitting procedures that are implemented in future improvements of these mass models and discuss how this may be achieved. We also show how certain neutron-proton correlations not taken into account in the GK approach may be incorporated so as to bring it into even closer agreement with measured masses throughout the periodic table.

The GK mass relations arise by imposing the condition that the various interactions between nucleons (neutronneutron, neutron-proton and proton-proton) cancel to first order. This gives rise to a series of mass relations between neighboring nuclei. The most commonly used relations involve three sets of mass differences, the first two of which can be written as

$$
\begin{aligned}
& M(N+2, Z-2)-M(N, Z) \\
+ & M(N, Z-1)-M(N+1, Z-2) \\
+ & M(N+1, Z)-M(N+2, Z-1)=0
\end{aligned}
$$

and

$$
\begin{aligned}
& M(N+2, Z)-M(N, Z-2) \\
+ & M(N+1, Z-2)-M(N+2, Z-1) \\
+ & M(N, Z-1)-M(N+1, Z)=0
\end{aligned}
$$

The first relation should only be applied when $N \geq Z$, to avoid situations in which a crossing of the $N=Z$ line leads to an improper cancellation of the isospin dependence of the residual interaction. When $N<Z$, the alternative relation

$$
\begin{aligned}
& M(N-2, Z+2)-M(N, Z) \\
+ & M(N-1, Z)-M(N-2, Z+1) \\
+ & M(N, Z+1)-M(N-1, Z+2)=0
\end{aligned}
$$

should be used. Also, these relations should not be used when odd-odd nuclei with $N=Z$ appear in the set of masses that are related, as for such nuclei the Wigner effect precludes accuracy to better than $1 \mathrm{MeV}$. Earlier 
estimates of the validity of the GK mass relations suggested that they are satisfied to roughly $180-200 \mathrm{keV}$ on average over the available experimental masses [4].

We have carried out a more thorough and systematic test of the accuracy of these mass relations for known nuclear masses, using the following algorithm:

1. Focussing on a given nucleus with $N$ neutrons and $Z$ protons, we identify all GK mass relations that contain the mass of that nucleus.

2. From each relation containing that nucleus, we deduce an estimate of its mass as a function of the masses of its various neighbors. From Eqs. (1-3), there are a total of 12 estimates possible, assuming all neighboring masses are available. For a given nucleus, in which $n$ estimates are possible $(1 \leq n \leq 12)$, we then calculate the mean value. We will from now on refer to this mean value as the GK prediction for the mass of the nucleus.

3. We then calculate the deviation between the measured mass of the nucleus and the GK predicted mass. Figure 1 shows these deviations, as obtained from the 2003 tabulation of masses (AME03) by Audi et al. [5].

4. Finally, we calculate the root-mean-square (rms) deviation of the predicted mass from the measured mass for all nuclei for which the same or a higher number $n$ of estimates are possible. The calculations are carried out for all nuclei with $A \geq 16$ and separately for all nuclei with $A \geq 60$, with the results presented in Table $\mathrm{I}$.

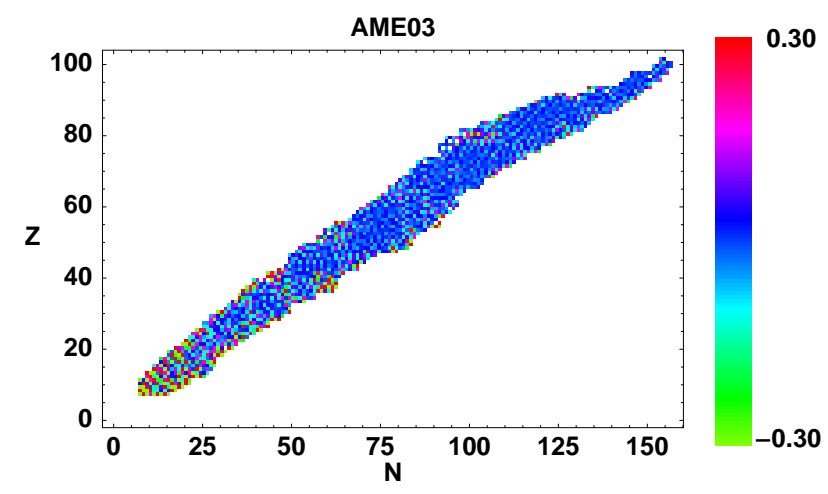

FIG. 1: Deviations (color coded) between the GK mass predictions and the measured masses.

The first point to note from the figure is the absence of any correlations in $N$ and $Z$ of the deviations between the measured masses and those estimated from the GK relations. This suggests that the deviations are simply white noise. Second, we see that across the entire periodic table there are very few nuclei, mostly in the light-mass
TABLE I: The rms deviations for masses (in $\mathrm{keV}$ ) calculated with the GK relations for different $n$.

\begin{tabular}{ccccc}
\hline \hline & $n \geq 1$ & $n \geq 4$ & $n \geq 7$ & $n=12$ \\
\hline$A \geq 16$ & 182 & 152 & 123 & 87 \\
$A \geq 60$ & 115 & 98 & 86 & 76 \\
\hline \hline
\end{tabular}

region, for which the deviations exceed $300 \mathrm{keV}$ (the red points). We may conclude that the GK relations provide an excellent test for the full range of known nuclear masses. The figure also indicates which masses are least well predicted by the GK relations, a point to which we will return later.

From Table I we see that the predictions of nuclear masses provided by the GK relations become increasingly more reliable as more estimates are possible. Clearly the greater the number of estimates possible the greater the number of experimental masses being sampled to pin down the mass of interest. In particular, when 12 estimates are possible, the set of GK mass relations can be combined into a single relation that contains 21 masses, namely

$$
\begin{aligned}
& M(N-2, Z+2)+M(N-2, Z-2) \\
+ & M(N+2, Z+2)+M(N+2, Z-2) \\
- & 2 M(N+2, Z-1)-2 M(N+2, Z+1) \\
- & 2 M(N-2, Z-1)-2 M(N-2, Z+1) \\
- & 2 M(N-1, Z-2)-2 M(N+1, Z-2) \\
- & 2 M(N-1, Z+2)-2 M(N+1, Z+2) \\
+ & 2 M(N+2, Z)+2 M(N-2, Z) \\
+ & 2 M(N, Z-2)+2 M(N, Z+2) \\
+ & 4 M(N+1, Z)+4 M(N-1, Z) \\
+ & 4 M(N, Z-1)+4 M(N, Z+1) \\
- & 12 M(N, Z)=0 .
\end{aligned}
$$

Thus, information on twenty neighboring masses will be used to pin down the GK predicted mass in such cases.

Indeed, in those cases for which 12 estimates are possible, the rms deviation for all nuclei with $A \geq 16$ is just $87 \mathrm{keV}$, whereas for nuclei with $A \geq 60$ it improves further to $76 \mathrm{keV}$. These results suggest that the GK relations can be applied reliably for interpolating between known masses. As such, they can be used to make very accurate predictions of unmeasured masses for nuclei that are near known regions.

We have also gauged the validity of the GK relations as we move away from the stability region. We divided the nuclear mass chart into five regions according to the distance $d$ to the stability curve, which is defined by the relation

$$
Z=\frac{2 A}{4+\alpha A^{2 / 3}}
$$

where $\mathrm{A}$ is the mass number and $\alpha$ is the quotient between the Coulomb and asymmetry parameters which 
TABLE II: The rms deviations for masses (in keV) calculated with the GK relations for different $n$ in different regions.

\begin{tabular}{ccccc}
\hline \hline Region & $n \geq 1$ & $n \geq 4$ & $n \geq 7$ & $n=12$ \\
\hline $0 \leq d \leq 2$ & 167 & 156 & 130 & 91 \\
$2 \leq d \leq 4$ & 207 & 154 & 121 & 76 \\
$4 \leq d \leq 6$ & 187 & 106 & 82 & 66 \\
$6 \leq d \leq 8$ & 106 & 103 & 93 & 98 \\
$8 \leq d \leq 10$ & 127 & 155 & 75 & \\
\hline \hline
\end{tabular}

can be obtained from a least square fit of the experimental masses to a liquid drop formula. In Table II we present the rms deviations between the experimental masses and the GK predictions for the five regions. From this table we see that the GK predictions are satisfied roughly equally in the various regions, especially for those cases in which $n$ is large enough. This suggests that the GK relations most likely do not become less precise as we approach regions far from stability.

Underlying the GK relations are two assumptions: (i) that the nucleus has a dominant independent-particle structure, and ( $i i)$ that the bulk of the residual interaction is primarily between nucleons in the same level. These assumptions are optimally satisfied near closed shells, explaining why the GK relations are able to accurately reproduce the large discontinuities in masses that arise for such nuclei.

The fact that the GK mass relations are so accurately satisfied across the periodic table suggests that they can also be used to test the consistency of a given nuclear mass model. In Table III, we consider the predictability of three of the currently preferred mass models, the Finite Range Liquid Drop Model (FRDM) [6], the DufloZuker (DZ) model [7]), and the mass model derived from Hartree-Fock-Bogolyubov theory [8], and show the rms deviations of the different mass models from the GK predictions calculated with eq.(4). We restrict the analysis to cases in which 12 estimates are possible. The results are shown for two data sets. The columns denoted 'All nuclei' refer to the results for all nuclei that are stable against neutron and proton emission according to the various mass models (i.e., up to the 'driplines'). The columns denoted 'AME03' refer to results for only those nuclei in the AME03 mass tabulation, as discussed earlier. Both the number of nuclei in the data set and the corresponding rms deviation from the GK predictions are quoted for each model. As can be seen from the table, all methods satisfy the GK relations quite well when restricted to the AME03 data set. Both the FRDM and HFB models, however, fail to satisfy the GK relations when extended beyond these nuclei. There is a nearly threefold loss in accuracy, a remarkable change. Considering how well the GK relations are satisfied throughout the known periodic table, these results suggest a lack of consistency with these relations in FRDM and HFB calculations, when extrapolated to unmeasured mass regions.

In contrast, the DZ mass table works well in reproduc-
TABLE III: The rms deviations (in keV) from GK relations with $n=12$ for the FRDM, DZ, and HFB models. The results are shown both for nuclei in the AME03 tabulation and for the full set of (predicted) particle-stable nuclei.

\begin{tabular}{rcccc}
\hline \hline Model & \multicolumn{2}{c}{ All nuclei } & \multicolumn{2}{c}{ AME03 } \\
& $\#$ & rms & $\#$ & rms \\
\hline DZ & 7431 & 44 & 1007 & 36 \\
FRDM & 7223 & 280 & 1007 & 103 \\
HFB & 7296 & 343 & 1007 & 138 \\
\hline \hline
\end{tabular}

ing the GK relations associated with the 2003 mass table and the more complete set of masses. It should be reiterated, however, that the DZ relations are based on similar shell-model assumptions as the GK relations and thus are inherently GK-compatible. Our results confirm that this GK-compatibility extends to previously unknown nuclei.

In Fig. 2 we present results for the same three mass models, but now comparing their deviations with respect to the GK predictions for individual nuclei. The analysis is carried out for the data sets referred to as "All Nuclei" in Table III. The red points refer to nuclei for which the deviation is 0.30 or greater, whereas the green points refer to nuclei for which they are -0.3 or less. As can be readily seen, the three figures are quite different from one another and, more significantly, very different from Fig. 1, which as a reminder displays the deviations between the GK predictions and the experimental masses that were available in 2003. Perhaps most notably, the deviations associated with the three mass models show clear correlations as a function of $N$ and $Z$, which, as noted before, are not observed in the experimental masses. This would seem to suggest that the three mass models, albeit perhaps in different ways, do not incorporate some important physical ingredients.

Despite the high level of accuracy and the associated predictability of the GK relations, there are some limitations. These show up in red in Fig. 1 as having larger than usual deviations between the GK estimates and the experimental masses and typically arise for odd-odd nuclei.

To confirm whether this is systematically the case, we have broken down the rms deviations in the GK calculations to those that derive from o-o, e-o, o-e and e-e nuclei, respectively. What we find is that the contribution to the rms deviation is largest for odd-odd nuclei. Restricting to those cases for which 12 estimates are possible, we find that the percentage contributions to the GK rms deviations are $35 \%, 23.7 \%, 25.6 \%$ and $15.7 \%$, for o-o, e-o, o-e and e-e, respectively. A possible way to qualitatively understand this is that the GK relations depend on having a simple independent-particle description of the ground state and such a description is best realized when there is little contamination in the ground state due to mixing of other configurations with the same $J^{\pi}$. In odd-odd nuclei, such contamination is expected to be largest.

This idea in fact provides the basis of some work by Zaochun et al. [9], who noted that for precisely this reason 

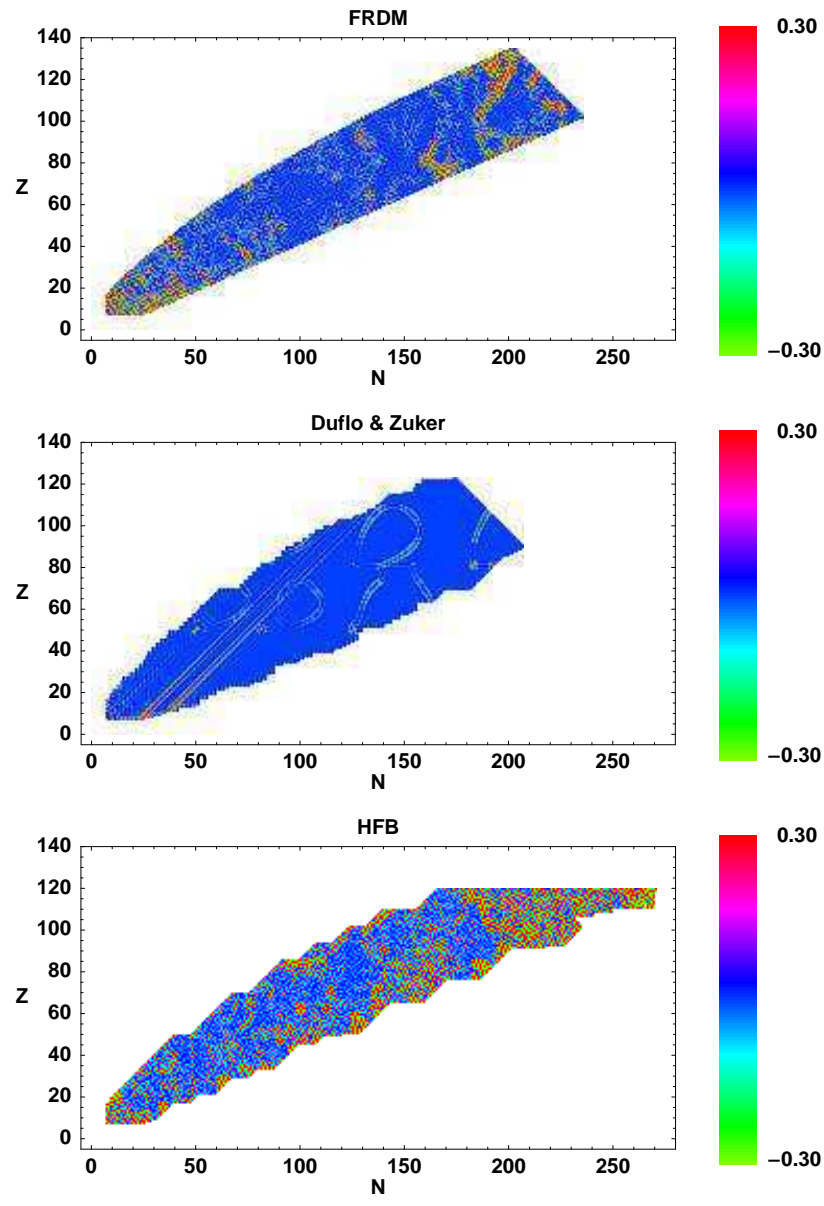

FIG. 2: Deviations (color coded) between the masses calculated using the mass models (a) FRDM, (b) Duflo-Zuker and (c) HFB and the GK estimates obtained from the neighboring masses. Red refers to nuclei with deviations or 0.30 or greater, green to nuclei for which they are -0.30 or less.

there is an effective enhancement of the neutron-proton interaction energy for odd-odd nuclei compared to oddmass or even-even nuclei. Their key point is that the neutron-proton interaction can induce perturbative correlations in the ground state of the system by mixing excited configurations through a term

$$
\Delta V_{\mathrm{np}}=\sum_{i} \frac{\left|\left\langle i\left|V_{\mathrm{np}}\right| \mathrm{gs}\right\rangle\right|^{2}}{E_{i}-E_{\mathrm{gs}}}
$$

where $|g s\rangle$ is the unperturbed ground state and $|i\rangle$ are unperturbed excitations. In even-even and odd-mass nuclei there is typically a large gap between the energies of the ground state and any state with the same $J^{\pi}$ as the ground state and such correlation effects are thus very weak. In odd-odd nuclei, this is not the case and one can have an enhancement of neutron-proton correlations.

Zhaochun et al. further show that in the absence of such perturbative corrections to the neutron-proton in- teraction one recovers the GK mass relations. This suggests that if one were to supplement the GK relations for odd-odd nuclei by removing such perturbative renormalization contributions due to the neutron-proton interaction one would arrive at modified GK relations able to achieve an even more accurate overall description of all nuclear masses. This should be explored in the future.

Friedman and Bertsch [10] have recently discussed some of the same issues from a slightly different perspective. They note that in odd-odd nuclei there is a dramatically increased degeneracy near the ground state and this leads to the need for an averaging over the possible neutron-proton couplings that can contribute. They refer to this as the odd-odd recoupling effect and note that it could most likely be accommodated in Density Functional Theory by including angular momentum projection. The precise connection between these ideas and those of Zhaochun and coworkers would be of interest to explore further, as would other plausible explanations as to why the GK relations are least well satisfied in oddodd nuclei.

The fact that the current mass formulae derived either from macroscopic/microscopic or purely microscopic considerations do not typically satisfy the GK relations when extrapolated to unmeasured masses, suggests that it might be of interest to modify these formulations to explicitly include the GK constraints. As an example, HFB treatments could include a fit to the GK relations in unknown regions in their search for parameters to use in the global analysis. Defining the calculated mass for a given nucleus to be $M_{\text {calc }}(N, Z)$ and the associated experimental mass to be $M_{\exp }(N, Z)$, the usual procedure is to determine the parameters of the global hamiltonian by minimizing $\sum_{N, Z}\left|M_{\text {calc }}(N, Z)-M_{\exp }(N, Z)\right|^{2}$ with the summation extending over all nuclei for which masses are known. We will now discuss how the quantity to be minimized might be modified so as to accommodate the simultaneous incorporation of the GK relations.

To do so, we denote a GK relation that centers on the mass of a nucleus with $N$ neutrons and $Z$ protons as $f(N, Z)=0$. We thus have in mind minimizing the functional (in $\mathrm{MeV}$ )

$$
\sum_{N, Z}\left|M_{\text {calc }}(N, Z)-M_{\text {exp }}(N, Z)\right|^{2}+\lambda \sum_{N, Z} g\left(f_{\text {calc }}(N, Z)\right),
$$

where $g(x)=|x|-0.1$, if $|x|>0.1$, and $g(x)=0$, if $|x|<0.1$. To the standard first term, which involves a sum over known masses, we add a second term which involves the calculated discrepancies of the GK relation, either for all nuclei or just for those for which experimental masses are unknown. Note that we have adopted a value of $0.1 \mathrm{MeV}$ as a target rms deviation that the GK procedure should realize, since this is approximately the value for known experimental masses and agreement to better than this level is thus meaningless. The coefficient of the second term $\lambda$ reflects the relative importance placed on the two terms in the functional. Ideally, we 
would like $\lambda$ small enough so that a good reproduction of the known masses is retained but at the same time large enough to permit an acceptable preservation of the GK relations throughout the periodic table. In the calculation of $f_{\text {calc }}(N, Z)$, we would use the $n=12$ estimate (cf. equation (4)). A more detailed presentation of these ideas will be published elsewhere [11].

While the above discussion has been centered on purely microscopic HFB-based treatments of masses [8], it could also be used in other theoretical studies of masses in which an effective hamiltonian is obtained parametrically. This suggests that it might be interesting to consider incorporating the GK relations in fits to determine the effective shell-model matrix elements, following the ideas presented above.

In summary, we have shown in this Letter that the GK mass relations may provide a valuable tool for improved systematic calculation of nuclear masses. We have found that the GK relations are satisfied to a very high level of accuracy by known masses, with an rms devia- tion of $87 \mathrm{keV}$ when all 12 estimates are possible, with the residual deviations having no discernible remaining correlations. We have also remarked that the accuracy with which the GK relations are satisfied does not show any evidence of decreasing when the nuclei in question get further away from the stability region. We have seen that the well-known mass formulae obtained either from macroscopic/microscopic or from purely microscopic considerations exhibit systematic patterns of deviation from the GK mass estimates, suggesting that some significant physical features may still be missing from them. We have thus suggested the possible use of the GK relations as a way to constrain and improve the calculation of nuclear masses.

This work was supported by Conacyt, Mexico, DGAPA and UNAM and by the US National Science Foundation under grant \# PHY-0553127. The authors also wish to express their appreciation to Andres Zuker for many helpful suggestions regarding this manuscript.
[1] C.E. Rolfs and W.S. Rodney, Cauldrons in the Cosmos (University of Chicago Press, Chicago, 1988).

[2] D. Lunney, J.M. Pearson, and C. Thibault, Rev. Mod. Phys. 75, 1021 (2003).

[3] G.T. Garvey, W.J. Gerace, R.L. Jaffe, I. Talmi, and I. Kelson, Rev. Mod. Phys. 41, S1 (1969).

[4] J. Barea, A. Frank, J.G. Hirsch, and P. Van Isacker, Phys. Rev. Lett. 94, 102501 (2005).

[5] G. Audi, A.H. Wapstra, and C. Thibault, Nucl. Phys. A 729, 337 (2003).

[6] P. Möller, J.R. Nix, W.D. Myers, and W.J. Swiatecki, Atom. Data and Nucl. Data Tables 59, 185 (1995).

[7] J. Duflo, Nucl. Phys. A 576, 29 (1994); J. Duflo and A.
P. Zuker, Phys. Rev. C 52, R23 (1995).

[8] S. Goriely, M. Samyn, P.-H. Heenen, J.M. Pearson, and F. Tondeur, Phys. Rev. C 66, 024326 (2002); M. Samyn, S. Goriely, P.-H. Heenen, J.M. Pearson, and F. Tondeur, Nucl. Phys. A 700, 142 (2002).

[9] G. Zaochun and Y.S. Chen, Phys. Rev. C 59, 735 (1999); G. Zao-chun, C. Yong-Zhou, and M. Jie, Chin. Phys. Lett. 18, 1186 (2001)

[10] W. A. Friedman and G. F. Bertsch, LANL preprint \# nucl-th/0702070 (2007).

[11] J. Barea et al.,to be published. 